\title{
Test of association of morphological variation with heterozygosity in the snail Cepaea nemoralis
}

\author{
L. M. COOK* \& G. GAO† \\ School of Biological Sciences, University of Manchester, Manchester M13 9PL, U.K.
}

\begin{abstract}
It has been suggested that the polymorphism for shell colour and pattern in Cepaea may be unrelated to the visible appearance of the shells, but maintained by heterosis or heterozygote advantage. A possible way to test this supposition is to examine whether phenotypic variance is lower in the morph exhibiting the dominant phenotype than in that with the recessive phenotype. This has been tested in two samples of shells involving totals of 1792 and 5423 individuals, respectively. Overall, there is a significantly greater variance in the recessive than the dominant class $(P<0.05)$. Correlation between measurements on the same shell was also examined. No evidence of difference between phenotypes was found. If the difference in variance is real it may indicate either: (i) that visible appearance is not important and the polymorphism is maintained by a heterozygous balance; or (ii) evolution of such a balance in a polymorphism maintained over a long time span by selection acting on visible attributes of the shells.
\end{abstract}

Keywords: Cepaea, heterozygosity, phenotypic variance, polymorphism, relational balance.

\section{Introduction}

The helicid land snails Cepaea nemoralis and $C$. hortensis are widespread in Europe and live in a wide range of habitat types from stony outcrops on mountain sides to sand dunes through mixed deciduous woodland, hedgerows and grassland. Most colonies are polymorphic for shell colour and banding, controlled by alleles which exhibit complete dominance. The two main loci, determining shell colour and the presence or absence of bands, are closely linked to each other and to a number of loci modifying band expression (Cook, 1967). Although morph frequencies may vary over short distances, there are hardly any colonies which do not have at least one morph at a frequency between 10 and 90 per cent. There is frequently linkage disequilibrium. Selection is definitely implicated in determining morph frequencies (Jones et al., 1977; Clarke et al., 1978; Cain, 1983), but the way in which the polymorphisms are maintained is not understood.

Although selection is related to the visible colours and patterns, with evidence of additional thermal

\footnotetext{
*Correspondence and present address: The Manchester Museum, University of Manchester, Manchester M13 9PL, U.K. †Present address: Biology Department, Shanxi Educational College, Taiyuan, Shanxi, China.
}

differences between morphs consequent on colour (Jones et al., 1977; Jones, 1982), it is possible that populations remain polymorphic because of heterozygote advantage or heterosis operating in some kind of nonvisible way. An advocate of this view is Goodhart (1987). He argued that most land snails are probably genetically heterogeneous as a result of balancing selection. If the genes involved have visible effects which are deleterious, their visible expression is suppressed, as in some other helicid snails of fairly uniform appearance, but if colours and patterns are essentially nonadaptive they simply flag up the underlying balanced polymorphism. Cepaea nemoralis and $C$. hortensis fall into the latter category, according to Goodhart.

If so, then to understand the polymorphism we should have to find a way of testing for heterosis. A method which has been used extensively is to measure the amount of polymorphism at enzyme loci in samples with different levels of phenotypic variability (e.g. Mitton \& Grant, 1984; Palmer \& Strobeck, 1986; Zouros, 1987). The prediction is that lower morphological variance and decreased asymmetry will be associated with greater genomic heterozygosity. Numerous studies on bivalve molluscs have investigated this relationship. Heterozygous individuals have been shown to grow faster, 
live longer and have more efficient protein synthesis than homozygous individuals (Beaumont, 1991). When there is a positive association of heterozygosity with growth rate there is often also a negative association with variance of size (Zouros \& Foltz, 1987; Koehn et al., 1988). If the association were the result of heterozygote advantage it should hold even if a single polymorphic locus is involved (Yezerinac et al., 1992). Some data can be interpreted as indicating heterozygote advantage (Koehn et al., 1988), whereas others have been interpreted as favouring relational overdominance, the effect of heterozygosity at an ensemble of linked loci (Zouros \& Foltz, 1987; Beaumont, 1991). If no association appears the question of a possible relation between variance and heterozygosity is open, other circumstances or other loci might display it, but an association does suggest some kind of genomic interaction. This approach could be used for Cepaea, but so far as we know, has not yet been attempted.

The existence of extensive collections of random samples of shells from polymorphic populations makes a related approach possible, namely to measure variance of shell characters in individuals with dominant morphs compared with those with recessive morphs. The dominant class consists of individuals which may be heterozygous or homozygous for the allele concerned and possibly for an extensive section of chromosome, while the recessive is homozygous for the allele and possibly for an equivalent chromosome section. If genomic heterozygosity is associated with lower phenotypic variance, then samples of shells of the dominant morphs should show smaller phenotypic variance than samples of recessive morphs from the same colony. Helicid snails grow to an adult shell size, at which a thickened lip is laid down on the shell and no further growth takes place. Measurements of adult shell dimensions therefore record any factors which have had an effect on shell growth during development. Foote \& Cowie (1988) made measurements on the helicid snail Theba pisana, which showed that developmental homeostasis operates. Heritability of shell dimensions measured as offspring-parent regression, has been recorded as about 0.6 for $C$. nemoralis (Cook, 1967) and 0.7 for a similarly shaped helicid, Arianta arbustorum (Cook, 1965).

\section{Materials and methods}

Two sets of $C$. nemoralis collections have been examined. The first of these consists of samples of individuals allowed to breed in enclosures to investigate environmental effects on morph frequency and shell size (Cook \& Cain, 1980). The colonies were monitored annually and all adult individuals which had been bred within the enclosures were collected, marked and scored for phenotype and the maximum shell diameter measured (to $0.1 \mathrm{~mm}$ ). As the density of the populations increased the mean size attained by adults went down, so that these individuals may have suffered some sort of competitive stress. Details of the ecology and the founder stocks are to be found in Cook \& Cain (1980). Variation in shell breadth has been examined in pink (the dominant class) and yellow individuals in 18 samples totalling 1792 individuals.

The second set comes from the extensive A. J. Cain collection, currently housed in the Manchester Museum. This consists of random samples collected by Cain and many workers associated with him over the past 45 years in studies of Cepaea polymorphism. Material collected by D. P. T. Burke $(1979,1989)$ was chosen for examination. Burke took a large number of samples from a restricted region in Ireland over a short period of time. Each sample was scored for colour (yellow or nonyellow being compared here) and banding (banded or unbanded, unbanded being dominant). On each shell the maximum breadth, height in the plane of the umbilicus and breadth of aperture were recorded (to $0.1 \mathrm{~mm}$ ). These dimensions are indicated in Fig. 1 . In all, 114 samples were examined, comprising 5423 individuals although not all could be included because of the small size in some of the samples of the smallest category to be compared. There was no difference in mean between classes within samples in either set of data.

For the Irish snails the mean frequency of the banded morph over samples was 0.740 with a standard deviation of 0.241 . The mean frequency of yellows was $0.403 \pm 0.285$. Using these morph frequency data and assuming Hardy-Weinberg genotype frequencies, we can estimate the average frequency of heterozygotes in the dominant classes for the two loci. The results are $0.905 \pm 0.110$ for the banded locus and $0.774 \pm 0.150$ for the colour locus. Either way, the dominant class contains a high frequency of heterozygotes.

Two types of comparison have been made between phenotypes. The first is the overall variability in the class. Variability in the dominant class should be lower than in the recessive class if genetic heterozygosity ensures that the growth pattern leading to final adult size and shape is better controlled. The second is correlation. Even if variances do not differ, one class may show greater correlation between dimensions. That is to say, indi- 


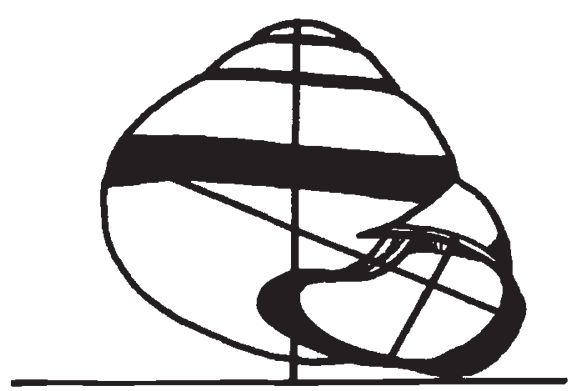

Fig. 1 Outline of the shell of Cepaea nemoralis showing the dimensions measured. The shell illustrated is midbanded, a genetically controlled variant of the five-banded phenotype, recessive to unbanded, which produces dark brown pigmented bands. The ground colour of the shell is yellow, pink or brown, these colours being controlled by an allelic series in which yellow is recessive to pink and brown, and pink is recessive to brown. There is more than one allele within each category, determining shades of colour. The banding and ground colour loci are closely linked. Typically, the lip to the aperture is brown in $C$. nemoralis but white in $C$. hortensis, but both species are sometimes polymorphic for this character.

viduals in one class may have a more uniform shape than those in the other. For snail shells, this measure includes the stochastic variation recorded as fluctuating asymmetry in studies of variables on the two sides of bilaterally symmetrical animals. Correlation has been examined between the three dimensions measured in the second data set.

When comparing variability between phenotypes, we have used the variance of breadth in the first data set and, in the second data set, the sum of variances for the three measurements. This is equivalent to the average sum of squares of the Cartesian distances of the values in each dimension from their joint mean (Van Valen, 1974). It is referred to as the total variance, as opposed to the generalized variance, which is the determinant of the covariance matrix for the variables. The intention is to compare variance in shell dimension in a class controlled by a dominant allele with that in the class controlled by the recessive allele. The comparison is between pink and yellow shells in the first data set and between either nonyellow and yellow or unbanded and banded in the second. For a single sample the most efficient way of carrying out a test is via the $F$ ratio. Between-sample variation is much greater than within-sample variation, so that results from individual $F$-tests for each sample should be combined. The samples are random ones, however, so that numbers in the classes are not controlled, the smaller category sometimes being much smaller than the larger. To include as many samples as possible, we have used all those in which the smaller category is five or more, and as a result, accidental effects of small sample size are likely to occur.

The $F$-test is usually a one-tailed test, the between-group variance being divided by the withingroup variance. In the present case a one-tailed test is appropriate, as the direction of the deviation is defined, but when the results from individual tests are combined, we should allow for the direction of deviation. In order to do so and to take account of variation in sample size, the following procedure has been used.

1 For each sample the larger variance has been divided by the smaller, and the direction of the difference has been scored.

2 The probability of obtaining the $F$-value has then been calculated, using a numerical algorithm to integrate the appropriate section of the $F$ distribution for the observed degrees of freedom (written for us by G. S. Mani; a FORTRAN program for the same purpose is given by Davies, 1971).

3 The equivalent normal deviate (the $t$-value for an infinite sample) is then substituted for the probability, using a second numerical estimate.

4 The deviates are made negative if the variance of the recessive class is smaller than that of the dominant class, otherwise they are made positive.

5 These values are then summed and divided by the square root of the number of deviates summed, to provide a single normal deviate measuring the tendency over all the data for the variance ratio to diverge from unity in a particular direction.

To examine correlation we have scored the mean of the three correlation coefficients $(r)$ for the variables in the second data set. The dominant class should show less variability. To test for this, the $r$-values have been converted to Hotelling's $z^{*}$, which has a variance of $1 /(n-1)$ where $n$ is the number in the class (Hotelling, 1953; Sokal \& Rohlf, 1969). Means for the two classes within samples have then been compared using the $t$-test. To detect a trend, the $t$-values have been converted to normal deviates and these combined as before to provide an overall measure of the tendency of one class to show greater variability than the other.

\section{Results}

The results for the data from the enclosure colonies are shown in Table $1 . F$-ratios are positive if the variance of yellow individuals is greater than that of pink individuals (the expected outcome if variance is 
Table 1 Variance ratios $(F)$ for shell breadth in two colour classes of Cepaea nemoralis, enclosure samples

\begin{tabular}{rrrrr}
\hline \multicolumn{1}{c}{$F$} & d.f. 1 & d.f. 2 & $P$ & \multicolumn{1}{c}{$d$} \\
\hline 1.289 & 77 & 290 & 0.140 & 1.475 \\
-1.260 & 257 & 43 & 0.359 & -0.917 \\
-1.206 & 66 & 24 & 0.615 & -0.500 \\
1.078 & 21 & 132 & 0.757 & 0.304 \\
1.350 & 21 & 110 & 0.313 & 1.008 \\
1.498 & 19 & 98 & 0.198 & 1.288 \\
1.332 & 16 & 68 & 0.399 & 0.842 \\
2.504 & 11 & 72 & 0.016 & 2.400 \\
1.678 & 11 & 63 & 0.186 & 1.322 \\
1.017 & 11 & 27 & 0.920 & 0.089 \\
-1.450 & 20 & 8 & 0.579 & -0.552 \\
-1.811 & 23 & 7 & 0.389 & -0.861 \\
-1.541 & 38 & 7 & 0.536 & -0.616 \\
-2.648 & 30 & 6 & 0.183 & -1.330 \\
3.531 & 82 & 5 & 0.113 & 1.584 \\
-1.829 & 44 & 5 & 0.460 & -0.737 \\
-1.130 & 18 & 4 & 0.980 & -0.011 \\
-1.130 & 18 & 4 & 0.980 & -0.011 \\
Overall & & & & 1.126 \\
\hline
\end{tabular}

The first column shows the $F$-ratio, made negative if the variance of the recessive class is smaller than that of the dominant class, otherwise positive. The next two are the degrees of freedom of the numerator and denominator, respectively, where the numerator is the dominant class if the variance ratio is negative, otherwise the recessive class.

$P$ is the probability corresponding to the variance ratio and $d$ is the equivalent normal deviate.

The samples have been arranged in descending order of sample size.

inversely related to genetic heterogeneity) and negative if yellows have the smaller variance. The last column gives the normal deviate equivalent to the $F$-ratio with the stated sample size. When these values are combined, the overall normal deviate is 1.126 , which is not significant. The same procedure has been used for the total variance of the three measurements in the second data set. The overall normal deviates for three different comparisons of classes, and the number of samples used to obtain them, are shown in Table 2. Comparing the colour classes, as before, the normal deviate is 1.732 in comparisons of 81 samples $(P<0.084$, two-tailed, 0.042 , one-tailed). When the second data set is classified by banding the equivalent figure is 1.448 in comparisons of 59 samples $(P<0.148$, two-tailed, 0.074 , one-tailed). It might be argued that the greatest difference should be seen by comparing yellow banded homozygotes with all phenotypes which are
Table 2 Overall normal deviates and probabilities for total variance in data from Irish Cepaea nemoralis

\begin{tabular}{lccc}
\hline Comparison & No. of samples & $d$ & $P$ \\
\hline Classified by colour & 81 & 1.732 & 0.042 \\
Classified by banding & 59 & 1.448 & 0.074 \\
Double recessive vs. others & 76 & 1.423 & 0.078 \\
\hline
\end{tabular}

Calculated in the same way as the overall normal deviate in Table 1. For each comparison, the dominant class has a smaller variance overall than the recessive class.

The double recessive class is yellow banded.

One-tailed probability is given.

not yellow banded. When this is done there are 76 samples available and the overall normal deviate is 1.423 , close to the figure for the banded/unbanded comparison. If the first data set and the second one classified by colour are combined, the overall normal deviate for this phenotype comparison is 2.021, which is significant $(P<0.043$, two-tailed, 0.022 , one-tailed).

For the whole data set, the correlation coefficient for breadth and height is 0.987 , that for breadth and mouth width is 0.988 and that for height and mouth width is 0.983 . Comparing the coefficients for the three measurements between unbanded and banded classes, there are 19 positive normal deviates in the 53 samples, five of them being significant. The deviate measuring the overall trend is 0.032 , however, indicating no tendency for one class to show greater correlation than the other.

\section{Discussion}

Populations of $C$. nemoralis are almost always polymorphic for shell colour and banding. Much has been discovered about the ways selection affects morph frequency (Jones et al., 1977; Clarke et al., 1978; Cain, 1983), but the question how the polymorphism is maintained has still not been answered. One line of reasoning suggests that as the polymorphism is visible the selection maintaining it is likely to involve visual predators, most probably exerting frequency-dependent selection (Clarke, 1969; Endler, 1988). This view is supported by analogy with other examples of adaptive coloration, such as mimicry in Lepidoptera, including mimetic polymorphisms (Clarke \& Sheppard, 1963, 1971; Sheppard, 1975; Turner, 1977). Even here, the universality of selective predation as the factor determining the patterns involved in mimicry may be questioned (Brower, 1995); and the reasons for the 
particular patterns present in land snail shells are not nearly as clear. The snails do not closely resemble particular elements of their environment (Clarke et al., 1978). In addition, in Cepaea it is reasonable to question whether such a labile controlling system as frequency-dependent predation could account for the apparent robustness of the polymorphism, where diversity is retained in such a wide range of habitats in populations of such variable size and in many cases in the apparent absence of predators. An alternative hypothesis was proposed by Goodhart (1987), that the visible aspect is part of a balanced combination of loci in which genomic heterozygosity is associated with high fitness. An analogy is to be found in the balanced inversion systems in Drosophila pseudoobscura studied by Dobzhansky and colleagues (e.g. Dobzhansky \& Levene, 1951, 1955; Dobzhansky \& Wallace, 1953) or with the supergenes controlling mimicry in the swallowtail butterflies Papilio dardanus and P. memnon (Clarke \& Sheppard, 1963, 1971). Recent considered opinion has been that supergenes such as the mimetic ones are exceptional (Maynard Smith, 1989), and Crow (1986) prefers to write instead 'in praise of multiple, independently inherited, additive genes'. Cepaea nemoralis is itself exceptional, however. The problem deserves to be resolved and the possibility that the visible polymorphism is part of a general heterozygosity system deserves attention. The present study was designed to test this proposition by looking for a negative association between phenotypic variance and scorable heterozygosity.

The overall deviations are positive in all cases and give some support to the suggestion that variance in shell dimensions is greater in the class recessive for a shell colour or pattern gene than in the one which is heterozygous or homozygous for the dominant. The combined result for colour is formally significant on both the one-tailed and two-tailed assumption, although not strongly so. On the other hand, there is no indication that correlation between dimensions differs between classes. The availability of good data sets in the form of shell collections tempted us to look for these associations, and the present result shows that further investigation would be worthwhile. However, to have good evidence that a positive deviation of the size observed indicates a true difference in variance between classes would require a total sample much larger than the present total of over 5000 individuals. One could then run into the problem of genetic heterogeneity between samples from different regions.

A greater variance of biometrical characters in the recessive class would not necessarily mean that the colours or patterns are nonadaptive. If the polymorphism has been present for a sufficiently long time, maintained perhaps by frequency-dependent selection acting on the visible phenotype (Clarke et al., 1978), there would be an opportunity for selection to operate on linked loci which increase heterosis. This type of process presumably accounts for the fact that Batesian mimetic butterflies, in which the visible characteristics are clearly adaptive in the presence of models, may also remain polymorphic in the absence of models. Examples of the complexity of the relation between the mimic/model system and the other selective forces involved are seen in studies of the Hypolimnas misippus/Danaus chrysippus complex (Edmunds, 1969; Smith 1976; VaneWright et al., 1977; Cook, 1978). Cepaea nemoralis and $C$. hortensis are species which have advanced northwards with the recession of the ice sheet during the Pleistocene. The greatest colour polymorphism is in the northern half of their range, and they are related to two southern species, $C$. sylvatica and $C$. vindobonensis, which are polymorphic for banding but not for colour. Whether the implied time span is long enough for the northern species to have developed a supergene system exhibiting heterozygote advantage is unknown.

\section{Acknowledgements}

We thank G. S. Mani for providing the method for obtaining probability of $F$ and A. J. Cain for helpful comments on the paper and methodology.

\section{References}

BEAUMONT, A. R. 1991. Genetic studies of laboratory reared mussels, Mytilus edulis: heterozygote deficiencies, heterozygosity and growth. Biol. J. Linn. Soc., 44, 273-285.

BROWER, A. V. Z. 1995. Locomotor mimicry in butterflies? A critical review of the evidence. Phil. Trans. R. Soc. B, 347, 413-425.

BURKE, D. P. T. 1979. Variation in Western Irish Populations of Cepaea nemoralis (L.). Ph.D. Thesis, University of Liverpool.

BURKE, D. Р. T. 1989. Variation in body colour in western Irish populations of Cepaea nemoralis (L.). Biol. J. Linn. Soc, , 36, 55-63.

CAIN, A. J. 1983. Ecology and ecogenetics of terrestrial molluscan populations. In: Russell-Hunter, W. D. (ed.) The Mollusca, vol. 6, Ecology, pp. 597-647. Academic Press, New York.

CLARKE, B. 1969. The evidence for apostatic selection. Heredity, 24, 347-353.

ClARKE, B. C., ARTHUR, W., HORSLEY, D. T. AND PARKIN, 
D. T. 1978. Genetic variation and natural selection in pulmonate molluscs. In: Fretter, V. and Peake, J. (eds) Pulmonates, vol. 2A, Systematics, Evolution and Ecology, pp. 219-270. Academic Press, New York.

ClARKE, C. A. AND SHEPPARD, P. M. 1963. Interactions between major genes and polygenes in the determination of the mimetic patterns of Papilio dardanus. Evolution, 17, 404-413.

ClaRKe, C. A. AND SHEPPARD, P. M. 1971. Further studies on the genetics of the mimetic butterfly Papilio memnon L. Phil. Trans. R. Soc. B, 263, 35-70.

CooK, L. M. 1965. Inheritance of shell size in the snail Arianta arbustorum. Evolution, 19, 86-94.

COOK, L. M. 1967. The genetics of Cepaea nemoralis. Heredity, 22, 397-410.

CooK, L. M. 1978. Zaire butterflies and faunal diversity in the tropics. Biol. J. Linn. Soc., 10, 349-360.

COOK, L. M. AND CAIN, A. J. 1980. Population dynamics, shell size and morph frequency in experimental populations of the snail Cepaea nemoralis (L.). Biol. J. Linn. Soc., 14, 259-292.

CROw, J. F. 1986. Basic Concepts in Population, Quantitative, and Evolutionary Genetics. Freeman, New York.

Davies, R. G. 1971. Computer Programming in Quantitative Biology. Academic Press, London.

DOBZHANSKY, TH. AND LEVENE, H. 1951. Development of heterosis through natural selection in experimental populations of Drosophila pseudoobscura. Am. Nat., 85, $247-264$.

DOBZHANSKY, TH. AND LEVENE, H. 1955. Genetics of natural populations. XXIV. Developmental homeostasis in natural populations of Drosophila pseudoobscura. Genetics, 40, 797-808.

DOBZHANSKY, TH. AND WALLACE, B. 1953. The genetics of homeostasis in Drosophila. Proc. Nat. Acad. Sci. Wash., 39, 162-171.

EDMUNDS, M. 1969. Polymorphism in the mimetic butterfly Hypolimnas misippus L. in Ghana. Heredity, 24, 281-302.

ENDLER, J. A. 1988. Frequency-dependent predation, crypsis and aposematic coloration. Phil. Trans. R. Soc B, 319, 505-523.

FOOTE, M. AND COWIE, R. H. 1988. Developmental buffering as a mechanism for stasis: evidence from the pulmonate Theba pisana. Evolution, 42, 396-399.

GOODHART, с. в. 1987 . Why are some snails visibly polymorphic, and others not? Biol. J. Linn. Soc., 31, 35-58.

HOTELLING, H. 1953. New light on the correlation coefficient and its transforms. J. R. Statist. Soc. B, 15,
$193-232$.

JONES, J. S. 1982. Genetic differences in individual behaviour associated with shell polymorphism in the snail Cepaea nemoralis. Nature, 298, 749-750.

JONES, J. S., LEITH, B. H. AND RAWLINGS, R. 1977. Polymorphism in Cepaea: a problem with too many solutions? Ann. Rev. Ecol. Syst., 8, 109-143.

KOEHN, R. K., DIEHL, w. J. AND SCOTT, т. M. 1988. The differential contribution of individual enzymes of glycolysis and protein catabolism to the relationship between heterozygosity and growth rate in the coot clam, Mulinia lateralis. Genetics, 118, 121-130.

MAYNARD SMITH, J. 1989. Evolutionary Genetics. Oxford University Press, Oxford.

MITTON, J. B. AND GRANT, M. C. 1984. Associations among protein heterozygosity, growth rate, and developmental homeostasis. Ann. Rev. Ecol. Syst., 15, 479-499.

PALMER, A. R. AND STROBECK, C. 1986. Fluctuating asymmetry: measurement, analysis, patterns. Ann. Rev. Ecol. Syst., 17, 391-421.

SHEPPARD, P. M. 1975. Natural Selection and Heredity, 4th edn. Hutchinson, London.

SMrTH, D. A. s. 1976. Phenotypic diversity, mimicry and natural selection in the African butterfly Hypolimnas misippus L. (Lepidoptera: Nymphalidae). Biol. J. Linn. Soc., 8, 183-204.

soKal, R. R. AND RoHlf, F. J. 1969. Biometry. The Principles and Practice of Statistics in Biological Research. Freeman, San Francisco.

TURNER, J. R. G. 1977. Butterfly mimicry: the genetical evolution of an adaptation. Evol. Biol., 10, 163-206.

VAN VALEN, L. 1974. Multivariate structural statistics in natural history. J. Theor. Biol., 45, 235-247.

VANE-WRIGHT, R. I., ACKERY, P. R. AND SMILES, R. L. 1977. The polymorphism, mimicry, and host plant relationships of Hypolimnas butterflies. Biol. J. Linn. Soc., 9, 285-297.

YEZERINAC, S. M., LOUGHEED, S. C. AND HANDFORD, P. 1992. Morphological variability and enzyme heterozygosity: individual and population level correlations. Evolution, 46, 1959-1964.

zoUROS, E. 1987. On the relation between heterozygosity and heterosis: an evaluation of the evidence from marine mollusks. Isozymes: Curr. Top. Biol. Med. Res., 15, 255-270.

zouros, E. AND FOLTZ, D. w. 1987. The use of allelic isozyme variation for the study of heterosis. Isozymes: Curr. Top. Biol. Med. Res., 13, 1-59. 